\title{
Review: Neuronal injury in HIV infection
}

\author{
CK Petito ${ }^{*, 1}$, AP Kerza-Kwiatecki ${ }^{2}$, HE Gendelman ${ }^{3}$, M McCarthy $^{4}$, A Nath ${ }^{5}$, ER Podack ${ }^{6}$, P Shapshak ${ }^{7}$ and \\ CA Wiley ${ }^{8}$ \\ ${ }^{1}$ Department of Pathology, 1550 NW 10th Avenue, PAP Bldg., Room 417, Miami, Florida, USA; ${ }^{2}$ National Institute of \\ Neurological Disorders \& Stroke, National Institutes of Health, Bethesda, Maryland, USA; ${ }^{3}$ Center for Neurovirology and \\ Neurodegenerative Disorders, University of Nebraska Medical Center Omaha, Nebraska, USA; ${ }^{4}$ Department of \\ Neurology, VA Medical Center Miami, Florida, USA; ${ }^{5}$ Department of Neurology, University of Kentucky, Lexington, \\ Kentucky, USA; ${ }^{6}$ Department of Microbiology \& Immunology, University of Miami, Miami, Florida, USA; ${ }^{7}$ Department of \\ Psychiatry, University of Miami, Miami, Florida, USA; ${ }^{8}$ Department of Pathology, University of Pittsburgh, Pittsburgh, \\ Pennsylvania, USA
}

Keywords: brain injury; microglia; monocytechemokine receptor; apoptosis; AIDS; HIV

The National Institutes of Neurological Disorders and Stroke (NINDS), a component of the National Institutes of Health (NIH), has a major commitment to the study of neurological disorders in patients infected by HIV. As a consequence, the NINDS has sponsored a series of symposia during which a small group of experts are invited to explore topics of specific importance to the field on neuroAIDS. 'The NINDS HIV Therapies Workshop' (1996) included a review of the natural history of neurological disease in HIV infection in the setting of available therapies and the challenge to develop improved treatments for peripheral and central nervous system disorders. The purpose of the NINDS AIDS Program panel: 'CNS as HIV Reservoir: BBB and Drug Delivery' (1998) was to bring together and to provide cross-fertilization of neuro-AIDS commmunity representatives and nationally and internationally recognized authorities on the blood-brain barrier and pharmacodynamics to discuss drug delivery to the nervous system and the central nervous system as the HIV sanctuary.

The current NINDS AIDS Progam panel, 'Neuronal Injury in HIV Infection' (1999), dedicated review sessions and discussions to neuronal injury and death in HIV infection, cell death mechanisms, signals of nerve cell injury, and clinical applications of the basic research. Neuronal injury underlies the clinical symptomatology of HIV-associated dementia (HIV-D) and is well documented in human disease, in animal models of HIV infection, and in cell culture experiments. This review

${ }^{*}$ Correspondence: CK Petito summarizes the presentations of the 'Neuronal Injury in HIV Infection' (1999) panel; lists its participants and invited guests; and includes the submitted abstracts, listed in alphabetical order. To provide continuity, this report is preceded by a short summary of the immediate past NINDS AIDS Program Panel (NAPP) held in 1998.

\section{NINDS AIDS Program Panel 'CNS as HIV reservoir' summary}

McArthur summarized the results of the first NAPP symposium, noting that the yearly incidence of HIV-D decreased in patients on highly active antiretroviral therapy (HAART) (triple therapy) when compared with patients treated with AZT alone or in combination with one other antiretroviral. Cerebrospinal fluid (CSF) viral load parallels the patients' therapeutic regimes: $80 \%$ of patients on triple therapy have undetectable loads compared with $25 \%$ of patients on monotherapy. Nevertheless, HIV-D remains a significant problem since only about one-third of AIDS patients in the United States are on HAART and since adherence to the strict drug regime is not always present or possible. Furthermore, the overall improved survival rate for patients on HAART may delay, rather than prevent, the eventual development of HIV-D. The cause and relevance of the previously documented blood-brain barrier (BBB) leak in post-mortem examination was examined and the potential for altering the BBB to improve penetration of some of the newer antiretroviral therapies was reviewed. The entire NAPP-1 summary has been published (AP Kerza-Kwiatecki, S Amini (1999). J Neurovirol 5: 113-114). 


\section{Neuronal injury in vivo}

Masliah presented data on selective vulnerability of neuronal injury in HIV infection. Specific neuronal characteristics that may impart vulnerability include the chemokine co-receptor profile; the concentration of chemokine and glutamate receptors; and the concentration of cytoplasmic calciumbinding proteins. The relative importance of these neuronal properties on conferring selective vulnerability (or resistance) in HIV infection may vary according to brain region.

Lackner discussed neuronal damage and the chemokine co-receptor profile in simian immunodeficiency virus (SIV) encephalitis, including studies showing that neuronal chemokine co-receptors are functional, and their activation increases intracellular calcium. Neuronal and synaptic damage in SIV encephalitis correlates with neuroinvasion and perivascular macrophages in brain. Of particular interest is the fact that decreases in the neuronal marker n-acetylaspartate (NAA), demonstrated by proton magnetic resonance spectroscopy (MRS), develop early after systemic SIV infection, but are reversible and do not become permanent until the evolution of macrophage-tropic virus.

Mucke and colleagues used transgenic mouse models to explore pathogenic mechanisms of HIV-related neuronal injury. He discussed the relationship between the systemic immune system and CNS degeneration in transgenic models in which macrophages, $\mathrm{T}$ cells and microglia express the human CD4 molecule. Stimulation of the peripheral immune system produces a CD4receptor-mediated neurodegeneration in this model. Mucke hypothesizes that this takes place via alterations in the profile of released microglial toxic factors.

\section{Apoptosis and programmed cell death}

Podack discussed the initiation of apoptosis by the interaction between specific ligands and cell surface death receptors, which leads to activation of the caspase cascade of proteases. Cytotoxic lymphocyte (CTL) killing of infected cells, including viral infections, provides exceptions to these events. These cells bypass the requirement for cell surface death receptors by secreting perforin, a protein that perforates and damages the target cell membrane. In an attempt at repair, the target cell endocytoses its damaged membrane but, in doing so, incorporates CTL-secreted proteases (granzymes) that themselves initiate the caspase cascade of apoptosis. HIV, among other viruses, possesses anti-apoptotic genes that interfere with CTL killing.

Boise reviewed the intracellular pathway of apoptosis concentrating on those steps mediated by the 14-member family of caspases. Some are activated during the interaction of cell surface death receptors whereas others have an effector role and are activated later in the caspase cascade. In certain conditions, a solitary cell death signaling agent such as TNF can activate two different caspase pathways, both of which need to be blocked in order to prevent apoptosis.

Dawson examined the primary role of poly (ADP Ribose) polymerase (PARP) activation in neurotoxicity. As stated in her abstract, PARP repair of nitric oxide-induced DNA breaks leads to cell death by depletion of cellular energy stores. Inhibition of PARP is neuroprotective, at least in certain model systems.

Wiley and co-workers examined the presence of DNA damage in HIV encephalitis by looking for the presence of DNA repair molecules, specifically choosing immunoreactivity for KU80, a protein component of DNA repair. They found increased KU80 expression in HIV encephalitis and a regional correlation between KU80 expression and diminished cortical-derived BDNF (brain-derived neurotropic factor). They suggest that withdrawal of normal neurotropic factors may be important in oxidative stress in HIVE. Related to Gendelman's presentation (see below) is Wiley's finding that the regional macrophages showed increased expression of BDNF.

\section{Macrophage-induced neurotoxicity}

Benveniste reviewed the similarities between activated microglia, the 'tissue monocyte' of the brain, and other tissue macrophages, with specific attention to the ability of certain cytokines to induce or suppress microglial expression of class II MHC antigen and co-stimulatory molecules. CD40 costimulatory molecule increases microglial expression of HIV-suppressive cytokines and decreases microglial expression of CXCR4. Although it would appear that CD40 expression would be beneficial, its cerebrospinal levels actually correlate with HIV-D, according to McArthur, and are increased in microglial nodules in SIV encephalitis, according to Lackner.

Gendelman discussed the potential for neurotoxicity to develop from the binding of an astrocyte secretory product SDF1 alpha to the CXCR4 cell surface receptors on neurons and astrocytes, thereby affecting intracellular signaling leading to apoptosis. He suggests that this interaction leads to decreased cAMP, calcium influx, neuronal hyperexcitability and eventual excitotoxic cell death. CXCR4 antibodies prevent SDF1 alphaassociated neuronal apoptosis in vitro. While it may seem paradoxical that SDF1 alpha mRNA is increased in HIV encephalitis, Gendelman hypothesizes that the activated macrophages might be both 
neuroprotective by releasing neurotropic factors as well as neurodestructive by releasing a variety of neurotoxins, some of which could bind to chemokine(s) receptors on neuronal cells.

Narayan described the macaque model of combined human-simian immunodeficiency virus infection (SHIV), in which the env portion of HIV1 is combined with the gag, pol, and other portions of SIV. In this model, productive SHIV encephalitis is species dependent, developing in rhesus macaques but not in pigtail macaques, although SHIV DNA is present in the brains of the pigtail macaques. Narayan suggests that IL4 is required for productive macrophage infection since Rhesus, but not pigtail, macaques retain the ability to produce Thy2 cytokines such as IL4. Administration of IL-4 enables brain macrophages of the pigtail macaques to sustain productive infection. He hypothesizes that the presence or absence of IL-4 may play a role in human disease and may underlie the difference between restricted versus productive infection.

\section{Virus or viral protein-induced neurotoxicity}

Sharer discussed the in vivo evidence that astrocytes harbor restricted infection by HIV with overexpression of regulatory genes and gene product, an observation first detected in pediatric AIDS brains and later documented in adults. Interestingly, Sharer found no evidence of restricted astrocyte infection in brains of macaques with SIV encephalitis. If in vitro restricted infection of astrocytes interferes with normal astrocytic function, Sharer hypothesizes that similar impairment could occur in vivo thereby interfering with neuronal function and stability.

McCarthy reviewed the evidence for restricted infection of astrocytes and neurons in vitro. In that setting, astrocyte infection shows a prediliction for lymphotropic strains of HIV-1. This characteristic of astrocytic infectivity is true following restricted infection by direct exposure to virus or following transfection with infectious proviral DNA.

Nath examined the ability of HIV gp120, Tat, Nef and Vpr to alter neuronal function when present in extracellular spaces. He pointed out that even transient exposure can cause neuronal dysfunction and that only certain subgroups of neurons in the culture dish will be affected - an in vitro example of selective vulnerability. Further, the neurotoxic effects of the viral proteins are synergistic. Cell surface molecules on target cells may mediate viral protein neurotoxicity. Nath has identified one such protein-on astrocytes (PAG: protein on astrocytes for gp120) that appears to play a role in gp120-induced increasees in intracellular calcium in astrocytes and gp120induced neurotoxicity.
Mattson also examined the neurotoxic effects of viral proteins (specifically Tat) and the intracellular signal pathways leading to Tat-induced neuronal apoptosis. Tat exposure in vivo elevates cytoplasmic free calcium which is followed by mitochondrial calcium uptake and secondary generation of oxygen free radicals and increases in the apoptosis-associated protein Par-4 (prostate apoptotic response-4). This pathway can be interrupted, or abolished, at multiple sites by calcium chelation, inhibition of mitochondrial calcium uptake, free radical scavengers, stabilization of mitochondrial membrane permeability and treatment with Par-4 antisense oligonucleotide. The signal for Tat-induced apoptosis is initiated at the synapse with activation of synaptic caspase 3 and propagation of the apoptotic signal to the neuronal perikaryon.

Lipton explored the mechanisms of gp120 neurotoxicity in mixed cultures of neurons, astrocytes and macrophages-microglia. Lipton and colleagues showed that inhibition of macrophage activation prevents gp120-induced neuronal apoptosis. They concluded that gp120 utilizes predominantly indirect pathways for neuronal injury, mediated by macrophage-released neurotoxins. Additionally, Lipton found that gp120 variants that preferred to bind to CXCR4 chemokine receptors (or were X4/R5 dual tropic) produced neuronal apoptosis, whereas beta-chemokines, including RANTES and MIP-beta, preferring to bind to CCR5, offered protection from gp120. In contrast, like gp120, the alpha-chemokines SDF-1alpha and beta, which bind to CXCR4 receptors, resulted in neuronal apoptosis. However, unlike gp120, the SDF-1 signaling appeared not to require macrophages but was mediated directly on neurons or astrocytes.

\section{Chemokine co-receptors}

Neuronal and glial chemokine co-receptors of HIV, which include CXCR4 (utilized by lymphotropic strains of HIV) and CCR5 (utilized by macrophagetropic strains), are potential mediators of CNS injury. Their importance was discussed in many of the presentations, as reviewed above.

Goldsmith reviewed the current status of chemokine-specific virus in the blood stream, pointing out that CCR5-specific viruses predominate during the early years of HIV infection whereas CXCR4-specific HIV may predominate in the late stages of AIDS. Work in his laboratory has shown that chemokine specificity directly correlates with target cell vulnerability and that a single point mutation in the HIV genome can change viral specificity for chemokine co-receptors. Of significance for neuropathogenesis are Goldsmith's findings that co-culture of target cells expressing either CD4 or CXCR4 permits HIVinduced infection or damage, raising the hypothesis 
that cell-to-cell contact of cells allows HIV infection of chemokine co-receptor positive, CD4-negative cells normally resistant to viral entry. This finding is supported by studies showing that HIV can infect CXCR4-positive astrocytes when co-cultured with CD4-positive lymphocytes.

\section{Viral co-infection}

Khalili and Wood discussed the deleterious effects of coinfection by JC virus or human herpesvirus-6 (HHV-6) respectively. Khalili and colleagues found that Tat-specific sequences bind to a specific nuclear protein (Pur-alpha); that Tat activates early and late JC virus promoters; and that oligodendroglial nuclei display Tat immunoreactivity, occurring perhaps via absorption of Tat from extracellular spaces. The Tat-Pur-alpha binding is functionally important in HIV and JCV replication in vitro and Khalili hypothesizes that a similar Tat-Pur-alpha interaction may be important in vivo.

Wood and McCarthy showed that co-infection by HHV-6 enhances HIV replication and that one of the HHV-6 genes so responsible also stimulates the induction of TNF-alpha; a cytokine that previously has been shown to also stimulate HIV replication. Of particular interest to CNS disease is the ability of HHV-6 to infect astrocytes and to enhance astrocyte replication of HIV.

\section{Abstracts}

\section{Microglial activation and inflammation}

Etty (Tika) Benveniste, Vince T Nguyen and

George M O’Keefe

Department of Cell Biology, The University of Alabama at Birmingham, Birmingham, Alabama 35294-0005, $U S A$

One of the characteristic features of microglia, the resident macrophage of the brain, is their rapid activation in response to injury, inflammation, neurodegeneration and infection. Activated microglia have functions similar to those of other tissue macrophages, including phagocytosis, antigen presentation, and production of cytokines/chemokines, eicosanoids, proteases and nitric oxide. Microglia are considered the main intrinsic immune effector cell of the central nervous system (CNS) due to their ability to express class II MHC antigens, as well as costimulatory molecules such as CD40 and B7.1/B7.2. We have conducted an analysis of the molecular basis of class II MHC and CD40 gene activation in microglia. IFN- $\gamma$ is the major inducer of both class II MHC and CD40, and there is an absolute requirement for the transcription factor STAT-1 in this response as microglia from STAT-1 deficient mice are unable to express class II MHC and CD40 in response to IFN- $\gamma$. IFN- $\gamma$ regulation of class II MHC expression first involves induction of the non-DNA binding transcription factor class II transactivator (CIITA). Analysis of the mouse type IV CIITA promoter revealed the involvement of GAS, E-box and IRF elements for IFN- $\gamma$ induction of this gene. Immunosuppressive cytokines such as TGF- $\beta$, IL- 4 and IL-10 inhibit IFN- $\gamma$ induced CIITA and class II MHC gene expression, and exert their effects at the level of CIITA transcription. Future studies are directed towards determining the molecular basis of TGF- $\beta$, IL-4 and IL-10 inhibition of CIITA promoter activity.
Regarding the CD40 promoter, regulatory elements located between -570 and $-456 \mathrm{bp}$ appear to mediate IFN- $\gamma$ induced activation. IL-4 and IL-10 also inhibit IFN- $\gamma$ induced CD40 expression by inhibiting transcription of the CD40 gene. Crosslinking of CD40 on microglia leads to induction of the chemokines RANTES, MIP- $1 \alpha$ (and MIP- $1 \beta$ and interestingly, to inhibition of CXCR4 receptor expression. Chemokine induction by this mechanism may be beneficial in diseases such as AIDS Dementia Complex. These studies highlight the role of the microglia as a multifunctional cell in the CNS.

\section{Regulation of caspase activation during apoptosis}

Lawrence H Boise

Department of Microbiology and Immunology, University of Miami School of Medicine, Miami, Florida, USA

Programmed cell death or apoptosis is a process that is essential for development of organisms as diverse as nematodes and mammals. While these organisms have been evolutionary distinct for several hundred million years, the mechanism by which they induce programmed cell death has been conserved. This mechanism ultimately results in the activation of a family of proteases referred to as caspases. Caspases are a distinct family of deathinducing cysteine proteases which cleave after aspartic acid. While all 14 mammalian caspases, which have been cloned, can induce cell death, the physiologic roles of these proteins fall into three classes. Several caspases such as Caspases 8 and 10 appear to be involved in the initial signaling of death and are referred to as 'upstream' or signaling caspases, while Caspases 3 and 7 play more of an effector role in cell death and are classified as 
'downstream' or effector caspases. A third subclass of caspases, including Caspase 1, appears to be more involved in the activation of inflammatory cytokines rather than in cell death. Caspase expression is not induced upon initiation of cell death, but rather these proteins exist as zymogens in all living cells. Thus death signals result in the activation of caspases, cleavage to active caspase by other proteases such as Granzyme B, or autoactivation of pro-caspases by multimerization. These mechanisms will be discussed in detail. Once activated, caspases cleave a variety of proteins are involved in the structure of the cell or may be involved in controlling various cell processes. The role of one of these substrates, Bcl-2, will be further discussed.

\section{Poly (ADP ribose) polymerase (PARP) in neurotoxicity}

Valina L Dawson

Department of Neurology, Johns Hopkins University

School of Medicine, Baltimore, Maryland, USA

Since we first described the critical role for nitric oxide (NO) in mediating glutamate neurotoxicity, we have continued investigating the regulation of NO synthase (NOS), targets of NO and conditions which lead to NO-mediated death in experimental models and human neurologic disease. Using pharmacologic approaches and genetic knockouts, we have clarified the role of NO as a neuronal messenger and neurotoxin. The primary cellular target for NO toxicity is the superoxide anion resulting in the generation of the potent oxidant, peroxynitrite, which can damage DNA leading to DNA nicks and breaks. The nuclear enzyme, poly (ADP-ribose) polymerase (PARP) senses these DNA breaks, and catalyzes the transfer of ADP ribose from NAD to nculear proteins such as histone and PARP itself. For every mole of ADP-ribose transferred by PARP, one mole of NAD and four free energy equivalents of ATP are consumed. Massive activation of PARP depletes NAD via ADP-ribose polymer formation. ATP is depleted in an effort to resynthesize NAD, leading to cell death by energy depletion. Inhibition of PARP activity spares the cell from energy loss, preventing irreversible depolarization of the neurons and thus providing neuroprotection. Consistent with this hypothesis, cortical cultures from PARP-null mice are dramatically resistant to NMDA, NO and combined oxygenglucose deprivation neurotoxicity. Infarct volumes are reduced $80 \%$ following transient middle cerebral artery occlusion in PARP-null mice as compared to wild-type littermate controls. Restoration of PARP activity in the PARP-null mice via viral vectors restores sensitivity to neurotoxicity. Addi- tionally, PARP-null mice are completely spared from the neurotoxic consequences of MPTP injection compared to significant dopaminergic cell loss in wild-type mice injected with MPTP.

While PARP cleavage is a classic biochemical marker for apoptosis, the role of PARP cleavage is unknown. Additionally, the mechanisms of PARP activation resulting in neuronal damage are under investigation. These initial results provide compelling evidence for a primary role for PARP activation in neuronal damage.

\section{Chemokines and their receptors in HIV-1-associated neural injury}

Jialin Zheng and Howard E Gendelman

The Center for Neurovirology and Neurodegenerative

Disorders and the Departments of Medicine and

Pathology and Microbiology, University of Nebraska

Medical Center, Omaha, Nebraska 68198-5215, USA

Chemokine receptors pivotal for HIV-1 infection in lymphocytes and certain ones (CCR3, CCR5, and CXCR4) are also expressed on neural cells including microglia, astrocytes and neurons. We theorized, therefore, that it is possible that any or all these viral co-receptors may mediate neural cell damage during HIV-1-associated dementia (HAD). This could occur without HIV-1 simultaneously affecting viral replication in neural cells. For example, CXCR4 serves as a mediator for neuronal apoptosis without eliciting HIV-1 infection. To these ends, we studied the role of diverse viral strains to affect intracellular signaling that may lead to neural (neurons, astrocytes) and monocyte-derived macrophages (MDM) apoptosis. Inhibitation of cAMP, activation of PI hydrolysis and apoptosis were observed by diverse HIV-1 strains principally in neurons. Virions recovered from lymphocytic strains (MN, IIB and Lai) produced the most significant effects in neuronal and astrocyte signaling and apoptosis. Macrophage tropic strains (ADA, JR-FL, Bal, MS-CSF and DJV) produced the least, while strain 89.6, a dual tropic HIV-1 strain, elicited intermediate neural cell damage. All lymphocytic strain's effects were blocked by the CXCR4 antibody, 12-G-5, while macrophage tropic strain's effect was only partially blocked by $12-\mathrm{G}-5$. This CXCR4 mediated neuronal apoptosis was confirmed in pure populations of rat cerebellar granule neurons. The HIV-1 envelope glycoprotein, gp120, induced markedly less neural damage seen with purified virions. Stromal-derived growth factor one alpha induced neuronal but not astrocyte or MDM apoptosis. Taken together, these results suggest that progeny HIV-1 virions can influence neuronal signal transduction and apoptosis through CXCR4. This process occurs independent of binding of CD4 and raises the possibility that lymphotropic lenti- 
viruses that use CXCR4 may play an important role in HAD.

\section{Coreceptors for HIV-1: Implications for pathogenesis and viral reservoirs}

Mark A Goldsmith

Gladstone Institute of Virology and Immunology,

University of California San Francisco, San Francisco,

California, USA

Human immunodeficiency virus-type 1 (HIV-1) primarily targets cells coexpressing CD4 in conjunction with an appropriate chemokine receptor, and the discovery of these coreceptors has markedly changed the paradigm by which AIDS pathogenesis is considered. CCR5 and CXCR4 have emerged as the predominant coreceptors for HIV-1 in vivo, whereas the contribution of other chemokine receptors has not yet been determined. CCR5specific (R5) viruses predominate during primary HIV-1 infection while viruses with expanded specificity for CXCR4 (R5/X4 or X4 viruses) often emerge in late stages of HIV disease. Marked changes in coreceptor preferences can be achieved through subtle changes in envelope sequence, including single point substitutions within the gp120 V3 loop (Speck et al, 1997). To explore possible relationships between viral tropism, coreceptor specificities and immune system destruction, we measured virus-induced CD4+ T-cell depletion in various systems including human lymphoid tissue cultured ex vivo. These cultures were inoculated with paired viruses that are genetically identical (isogenic) except for select envelope determinants specifying reciprocal tropism for CXCR4 or CCR5. In each system, X4 variants massively depleted CD4+ lymphocytes while matched R5 viruses caused only mild depletion despite comparable replication kinetics. These findings indicate that the coreceptor specificity of HIV-1 is a virulence factor, and that the evolution of viral envelope leading to usage of CXCR4 in vivo contributes directly to disease progression (Penn et al, 1999). Moreover, we found that this acceleration of pathogenesis can be abrogated by an antagonist of CXCR4, demonstrating that coreceptor engagement by the virus contributes to this process.

It is likewise speculated that pathogenesis in nonlymphoid tissues is influenced by coreceptor specificities. For example, in a recent functional survey based on cross-sectional samples we found evidence pointing to a central role for CCR5 in human brain infections, independent of development of dementia (Chan et al, 1999). In addition to effects involving CD4+ T-cells, HIV-1 infection of various CD4-negative cell types in the brain and elsewhere has been detected in vivo and in vitro, and these events may facilitate formation of viral reservoirs and/or be intrinsically pathogenic. The molecular basis of these infections (including a possible role for chemokine receptors) is unknown. We speculated that CD4 molecules displayed on neighboring cells may be provided to CD4-negative target cells in trans, which might permit triggering of virus-cell fusion. To test this hypothesis, we established a co-culture system in which various human CD4-positive T-cells lacking CCR5 are mixed with cells expressing CCR5 and challenged with HIV-1 bearing CCR5-dependent envelopes. Marked infection of the CCR5-positive targets was readily detected in the presence of CD4-bearing cells. Primary human astrocytes in culture were also susceptible target cells selectively in the presence of CD4+ cells. Collectively these findings reveal that CD4 and CCR5 need not be expresed together on target cell surfaces and can cooperate as an effective viral receptor complex in trans, thereby potentially expanding the range of susceptible viral targets for HIV-1. These findings together highlight the central and specific role of coreceptors in HIV-1 pathogenesis in the lymphoid compartment, central nervous system and elsewhere.

\section{References}

Chan SY, Speck RF, Power C, Gaffen SL, Chesebro B, Goldsmith MA (1999). V3 recombinants indicate a central role for CCR5 as a coreceptor in tissue infection by HIV-1. J Virol 73: $2350-2358$.

Penn ML, Grivel J-C, Schramm B, Goldsmith MA, Margolis L (1999). CXCR4 utilization is sufficient to trigger CD4+ T-cell depletion in HIV-1-infected human lymphoid tissue. Proc Natl Acad Sci USA 96: 663-668.

Speck RF, Wehrly K, Platt EJ, Atchison RE, Charo IF, Kabat D, Chesebro B, Goldsmith MA (1997). Individual amino acids within the HIV-1 envelope V3 region determine the utilization of specific chemokine coreceptors for cell entry. J Virol 71: 7136-7139.

\section{Molecular interaction of TAT and PUR $\alpha$ : An important regulatory event in replication of HIV-1 and JCV in CNS cells} Kamel Khalili ${ }^{1}$, Edward M Johnson ${ }^{2}$, Gary L Gallia ${ }^{1}$ and Jay Rappaport ${ }^{1}$

${ }^{1}$ Center for NeuroVirology and NeuroOncology, MCP Hahnemann University, 245 N. 15th Street, Mail Stop 406, Philadelphia, Pennsylvania 19102, USA;

${ }^{2}$ Department of Pathology, Mt. Sinai School of Medicine, Annenberg Building 1508, 1 Gustave L. Levy Place, New York, NY 10029, USA

HIV-1 infection induces a spectrum of clinical abnormalities affecting the central nervous system (CNS). These include behavioral, cognitive, as well as motor disturbances. These abnormalities are observed in at least two thirds of patients with AIDS. Histologically, brains of patients with HIV-1 
associated neurological disorders exhibit multifocal giant cell encephalitis (MGCE) and/or progressive diffuse leukoencephalopathy (PDL). Although microglia represent the major site for HIV-1 infection in the CNS, a broad range of histopathology including astrogliosis, cytolytic destruction of oligodendrocytes and demyelination, and apoptosis of neurons is also observed. These changes are believed to be mediated by paracrine mechanisms involving the secretion of host and viral factors which orchestrate the neurodegenerative disease process. One of the major mechanisms for AIDS pathogenesis in the CNS appears to be mediated by the HIV-1 regulatory protein, Tat, which can be secreted by infected cells, and taken up by noninfected cells, and activate gene expression by a paracrine pathway. This property of Tat permits perturbation of gene expression beyond the limits of foci of HIV-1 infection. Tat transactivates the HIV-1 LTR via an RNA element termed TAR at the $5^{\prime}$ end of all HIV-1 mRNAs. Tat also activates transcription of heterologous promoters including those of several cytokines as well as certain heterologous viral promoters. Notably, the late region promoter of JCV, the causative agent of the fatal demyelinating disease, Progressive Multifocal Leukoencephalopathy (PML), is responsive to Tat induction. The frequency of PML, a rare disorder seen in immunosuppressed individuals, is increased in the setting of HIV-1 infection, consistent with a functional role for Tat in PML pathogenesis of AIDS. Comparison of the JCV and HIV-1 promoters, and the study of the mechanism of Tat-mediated transactivation by our group over the last several years has identified a host protein, Pur $\alpha$, which mediates the association of HIV-1 Tat protein with both HIV-1 and JCV regulatory sequences. We provide evidence suggesting that the physical interaction of Pur $\alpha$ and Tat may become functionally important for the regulation of HIV-1 and JCV gene expression in CNS cell cultures. According to our biochemical data, interaction betwen Pur $\alpha$ and HIV-1 Tat is mediated by specific RNA molecules present in the cells. The region of Tat which is important for Pur $\alpha$ :Tat interaction includes the domain responsible for binding of Tat to HIV-1 TAR. A ten nucleotide GC-rich consensus sequence in RNAs associated with Pur $\alpha$ derived from human glial cells plays an important role in Pur $\alpha$ :Tat interaction, and that its overexpression in cells enhances Tat transcriptional activity in CNS cells. These observations provide an important clue regarding the pathways involved in Tat-induced activation of HIV-1, JCV, and other cellular gene expression.

\section{Early neuronal injury in SIV infected macaques: A potential role for chemokines and their receptors}

AA Lackner, S Klein, K Westmoreland, X Williams, A Alvarez, L Luster, L Becerra, E Cheng and R Masliah, Gonzalez R

Massachusetts General Hospital and New England Regional Primate Research Center, Harvard Medical School; University of California, San Diego, USA

Infection of rhesus macaques with SIV results in rapid neuroinvasion and neuropathologic abnormalities that are similar to those observed in HIVinfected humans. We have used this model to examine the role of chemokines and their receptors in neuroinvasion and the development of neuronal injury and neurologic disease. We previously demonstrated elevated expression of the chemokines MIP- $\alpha$, MIP- $1 \beta$, and RANTES and the corresponding receptors CCR3, CCR5, and also CXCR4 in perivascular infiltrates in the brain. Of additional interest was the presence of CCR3, CCR5, and CXCR4 on a subpopulation of large hippocampal and neocortical pyramidal neurons and on glial cells in both normal and SIV-infected brain. The expression of known HIV/SIV coreceptors on neurons and astrocytes suggested a possible mechanism whereby HIV/SIV or the chemokines they induce could interact with these cells, disrupting their normal physiologic function and contribute to the neuropathogenesis of AIDS. To address this hypothesis, we have used immediately ex vivo and in vitro techniques to confirm the presence of all three of these chemokine receptors on a subpopulation of neurons and CCR5 and CXCR4 expression on the majority of astrocytes. These chemokine receptors are functional as demonstrated by increased intracellular calcium in response to the appropriate ligand. We have also used proton magnetic resonance spectroscopy (MRS) to evaluate the neuronal marker n-acetylaspartate (NAA) both in vivo and ex vivo. A $25 \%$ decrease in NAA was detected 14 days after infection coincident with peak viremia, neuroinvasion and increased numbers of perivascular macrophages. Decreases in NAA were correlated with decreases in synaptophysin and calbindin in adjacent sections of brain. A further $10 \%$ decrease in NAA levels was observed in animals infected for 2 years or more regardless of the presence of SIVE. These results indicate that neuronal injury occurs early after viral infection, is associated with neuroinvasion and progresses during the course of infection. The interaction of chemokines or viral envelope with these functional chemokine receptors on neurons and astrocytes suggests a physiologic mechanism whereby neuronal injury could occur. 


\section{Glutamate- and chemokine-receptors in neuronal vulnerability}

Stuart A Lipton and Marcus Kaul

CNS Research Institute, Brigham and Women's Hospital, and Program in Neuroscience, Harvard Medical School,

Boston, Massachusetts 02115, USA

The number of HIV-1 infected cells in the brain is relatively small, and productively infected cells are exclusively of monocytoid lineage. This suggests that HIV-1 initiate a neurodegenerative process that entails amplification to produce pronounced CNS injury. Indeed, in culture systems of both rodent and human brain, HIV-1 infected or gp120-stimulated macrophages and microglial have been found to release neurotoxins that contribute to the neurodegenerative process, at least in part, by excessive stimulation of the $N$-methyl-D-aspartate (NDMA) subtype of glutamate receptor. The fact that gp120-transgenic mice manifest neuronal damage resembling that found both in rodent cultures exposed to gp120 and in human brains with AIDS dementia indicates that, even in the absence of intact HIV-1, a fragment of the virus is sufficient to trigger important aspects of this amplification cascade in the neurodegenerative process and therefore has relevance to in vivo pathogenicity.

In vitro and in vivo, either macrophage- or T-cell tropic variants of gp120 produce injury and apoptosis in both primary rodent and human neurons. Recent evidence has shown that gp120 binds respectively to macrophages and T-cells via the chemokine receptors CCR5 and CXCR4, which, in addition to CD4, function as co-receptors for HIV1. Nonetheless, these and other chemokine receptors are also present on and trigger signaling pathways in neurons and astrocytes. Thus, the question arises whether or not gp120-induced neuronal injury occurs as a consequence of direct interaction with neurons via chemokine receptors and their cognate-G protein signaling systems, or indirectly via the release of macrophage toxic factors, as previously suggested from in vitro experiments with gp120-conditioned medium after macrophage depletion. Finally, both pathways in conjunction could contribute to neuronal death, as recently shown for CD8+ T-cells. Although gp120 can signal via chemokine receptors on neuronal cell lines and neurons cultured in isolation, the propensity for cell-cell interactions mandates that disease pathogenesis in vitro must be approached in a 'mixed' culture that recapitulates the types and proportion of cells normally found in brain, i.e., neurons, astrocytes, and macrophages/microglia.

We have found in such a mixed culture system that the $\beta$-chemokines RANTES or MIP-1 $\beta$ can protect rodent cerebrocortical neurons from gp120-induced apoptosis. RANTES or MIP-1 $\beta$ (each at $20 \mathrm{nM}$ ) abrogated neuronal apoptosis induced by
$200 \mathrm{pM}$ recombinant gp120 $0_{\mathrm{SF} 2}$, whilst BSA $(0.001 \%=144 \mathrm{nM})$ and the $\alpha$-chemokines produced neurotoxicity on their own ( $\sim 2$ fold increase in neuronal apoptosis compared to control). MIP-1 $\beta$ and RANTES presumably inhibit the neurotoxic effect of $\mathrm{gp} 120_{\mathrm{SF} 2}$ in an indirect manner, since RANTES binds to the B-chemokine receptors CCR1, CCR3 and CCR5, and MIP-1 $\beta$ to CCR5 (or functional homologue), whereas gp120 $0_{\mathrm{SF} 2}$ and SDF$1 \alpha / \beta$ interact with the a-chemokine receptor CXCR4. Note that although gp120 $0_{\mathrm{SF} 2}$ may also interact to a lesser degree with the $\beta$-chemokine receptor CCR5 on transfected cell lines, this has not been shown to occur on primary cells, as used here. Moreover, rodent cerebrocortical cultures are a suitable model system to study these actions of gp120 since these species express CXCR4 homologues which, like the human CXCR4, are capable of mediating HIV-1 infection via gp120 binding.

These findings, however, do not tell us if the neuroprotective effect of the $\beta$-chemokines and, for that matter, the neurotoxic effect of gp120 is mediated by macrophages, astrocytes, neurons, or by simultaneous action on two or all three cell types. To answer this query, we used the macrophage inhibitory tri-peptide Thr-Lys-Pro (TKP), which specifically prevents the release of endogenously-synthesized macrophage factors in vitro and inhibits microglial activation in vivo in response to excitotoxins without killing the cells, whereas control peptides have no effect. In our experiments, TKP $(50 \mu \mathrm{M})$ protected neurons from gp120-induced apoptosis, similar to our previous experiments which depleted macrophages from the cultures. This finding indicates that activated macrophages are necessary for gp120-induced neuronal apoptosis if the various cell types represented in brain are present in the culture system. Hence, the predominant pathway to gp120-induced neruonal apoptosis appears to be mediated by the release of macrophage toxins, which lead to NMDA receptor-mediated damage.

\section{Patterns of neurodegeneration in HIV encephalitis}

E Masliah

Department of University of California, San Diego School of Medicine, La Jolla, California, USA

AIDS patients with HIVE show both cognitive and motor impairments. While the motor alterations might be related to basal ganglia damage, cognitive alterations could be associated with damage to limbic structures and frontal cortex. These brain regions contain diverse neuronal populations that are differentially susceptible to damage directly or indirectly mediated by HIV-1. Damage to neocortical neuronal populations includes neurons, which 
bear glutamate receptors and show low levels of calcium-binding proteins. Differences in the relative levels of glutamate receptors, calcium binding proteins and cytokine receptors across different neuronal populations may determine their selective vulnerability to distinct HIV induced neurotoxins. This postulate is supported by recent findings in the human brain, as well as in transgenic mice everexpressing gp120 and IL6. In HIVE there is a significant decrease in calbindin-immunoreactive pyramidal neurons and interneurons in the neocortex, while the numbers of calbindin-positive cells that appear to be resistant to neurodegeneration in HIVE are GABAnergic spiny neurons that also release dynorphin, substance $\mathrm{P}$ and enkephalin. In addition to the damage in the neocortex and basal ganglia, HIVE cases displayed hippocampal damage characterized by loss of interneurons. These regional differences in the patterns of selective damage to neurons in the HIVE brain suggest that in the neocortex neurons are more vulnerable to HIV-1 mediated damage, while in the hippocampus and basal ganglia cytokines might play a significant role. Other important factors to consider as mediators of selective neuronal damage includes the presence of different chemokine receptors in susceptible neurons.

\section{Acknowledgements}

This work was supported by NIH grant MH 45294 .

\section{Synaptic apoptosis, Par-4 and the pathogenesis of AIDS dementia}

MP Mattson, L Kruman, A Nath and JD Geiger

Sanders-Brown Research Center on Aging and

Department of Anatomy \& Neurobiology, University of

Kentucky, Lexington, Kentucky, USA

Our recent studies of molecular mechanisms of neuronal degeneration in Alzheimer's disease (AD) have provided evidence that synaptic compartments are sites where the neurodegenerative process is initiated, and that oxidative stress and perturbed calcium homeostasis in these compartments are central to the pathogenesis of $\mathrm{AD}$ (Mattson, 1997; Mattson et al, 1998; Guo et al, 1999). Many patients infected with HIV-1 exhibit cognitive deficits that are related to progressive neuronal degeneration and cell death. The protein Tat, which is released from HIV-1 infected cells, induces apoptosis in cultured embryonic rat hippocampal neurons (Kruman et al, 1999). Tat induces caspase activation, and the caspase inhibitor zVAD-fmk prevents Tat-induced neuronal death. Tat induced a progressive elevation of cytoplasmic free calcium levels which is followed by mitochondrial calcium uptake and generation of mitochondrial reactive oxygen species (ROS). The intracellular calcium chelator BAPTA-AM and the inhibitor of mitochondrial calcium uptake ruthenium red protected neurons against Tat-induced apoptosis. ZVAD-fmk suppressed Tat-induces increases of cytoplasmic calcium levels and mitochondrial ROS accumulation indicating roles for caspases in the perturbed calcium homeostasis and oxidative stress induced by Tat. An inhibitor of nitric oxide synthase, and the peroxynitrite scavenger uric acid, protected neurons against Tat-induced apoptosis indicating requirements for nitric oxide production and peroxynitrite formation in the cell death process. Tat causes a delayed and progressive mitochondrial membrane depolarization, and cyclosporin a prevented Tat-induced apoptosis, suggesting an important role for mitochondrial membrane permeability transition in Tat-induced apoptosis.

Levels of Par-4, a protein recently linked to neuronal apoptosis in AD (Guo et al, 1998), are increased in neurons in hippocampus of AIDS dementia patients and monkeys infected with a chimeric strain of HIV-1 and simian immunodeficiency virus. Par-4 levels increased rapidly in cultured hippocampal neurons following exposure to the neurotoxic HIV-1 protein Tat, and treatment of the cultures with a Par-4 antisense oligonucleotide protected the neurons against Tat-induced apoptosis. Additional findings show that Par-4 participates at an early stage of Tat-induced neuronal apoptosis prior to caspase activation, oxidative stress and mitochondrial dysfunction. Our data suggest that Par-4 may be a mediator of neuronal apoptosis in AIDS dementia.. Collectively, our data demonstrate that Tat can induce neuronal apoptosis by a mechanism involving disruption of calcium homeostasis, Par-4 induction, caspase activation, and mitochondrial calcium uptake and ROS accumulation. Agents that interrupt this apoptotic cascade may prove beneficial in preventing neuronal degeneration and associated dementia in AIDS patients.

\section{Summary of NAPP-1, September 1998}

Justin C McArthur

Department of Neurology, Johns Hopkins University School of Medicine, Baltimore, Maryland, USA

With the advent of highly active antiretroviral therapy (HAART), the incidence of HIV-associated dementia has declined by $40-60 \%$ in the US (Sacktor et al, 1999). A corollary to this, however, is that because the survival of individuals with AIDS is now so much longer (Palella et al, 1998), we anticipate the prevalence to rise. Fifteen to twenty per cent of those with AIDS will develop HIV-associated cognitive dysfunction. There are now 13 FDA-approved antiretrovirals available in 
the US. For many of them, particularly the potent protease inhibitors, point plasma:CSF ratios are low, which might predict poor CNS penetration. In fact, because of the low degree of protein binding within the CSF, free levels of some of those agents may in fact be adequate for durable virological suppression HAART may suppress the trafficking of infected cells, and there is now accumulating evidence that HAART regimens can improve neuropsychological performance and radiological abnormalities in HIV dementia (Sacktor et al, 1990; Ferrando et al, 1998).

Pathological studies in both HIV and SIV have demonstrated a perturbation of the blood-brain barrier (BBB) (Power et al, 1993). The timing of this, with respect to initial infection of the CNS, the mechanisms of its production, and its functional significance remain uncertain. Despite the pathological evidence, there is as yet, little radiological evidence from magnetic resonance imaging of the entry of gadolinium into the parenchyma, traditional the radiological marker of a perturbed BBB. CNS macrophage activation, which accompanies HIV encephalitis, is associated with the local release of $\beta$-chemokines, metalloproteinases, and the upregulation of endothelial adhesion markers. The interactions of these substances could lead to disturbance in the integrity of the blood-brain barrier (Conant et al, 1998). The consequence of this might be to facilitate the entry of infected monocytes and lymphocytes and, in addition, might also expose the brain to high levels of circulating neurotoxic substances, including cytokines and plasma constituents. In vivo systems modeling the blood-brain barrier have been developed and may lead to a better understanding of structure/function abnormalities in vivo.

\section{References}

Conant K, Garzinodemo A, Nath A, McArthur JC, Halliday W, Power C, Gallo RC, Major EO (1998). Induction of monocyte chemoattractant protein-1 in HIV-1 Tat-stimulated astrocytes and elevation in AIDS dementia. Proc Natl Acad Sci 95 $3117-3121$.

Fernando S, Rabkin J, van Gorp W, McElhiney M (1998). Protease inhibitors are associated with less neuropsychological impairment in HIV infection. 5th Conference on Retroviruses and Opportunistic Infections, in press.

Palella Jr FJ, Delaney KM, Moorman AC, Loveless MO, Fuhrer J, Satten GA, Aschman DJ, Holmberg SD (1998). Declining morbidity and mortality among patients with advanced human immunodeficiency virus infection. HIV Outpatient Study Investigators [see comments]. N Engl J Med 338: 853860.

Power C, Kong PA, Crawford TO, Wesselingh S, Glass JD, McAuthur JC, Trapp BD (1993). Cerebral white matter changes in HIV dementia: alterations in the blood-brain barrier. Ann Neurol 34: 339-350.

Sacktor NC, Skolasky RL, Esposito D, Lyles RH, McFarlane G Selnes OA, McArthur JC (1999). Combination antiretroviral therapy including protease inhibitors improves HIV-associated cognitive impairment. Neurol in press.
Sacktor NC, Lyles RH, McFarlane G, Skolasky RL, Kleeberger C, Becker JT, Cohen B, Wesch J, Miller EN, McAuthur JC, the Multicenter AIDS Cohort Study (1999). The changing incidence of HIV-1-related neurological diseases: 19901997. 6th Conference on Retroviruses and Opportunistic Infections, in press.

\section{Restricted HIV-1 infection of neurons and glia in vitro}

\section{McCarthy}

Department of Neurology, University of Miami School of Medicine Miami VA Medical Center Miami, Florida, USA

Neuroepithelial lineage cells (astrocytes, neurons, and oligodendrocytes) harbor relatively low but arguably significant levels of HIV-1 infection in vivo (Bagasra et al, 1996). Approximately 40-45\% of astrocytes or neurons with detectable HIV-1 DNA have some evidence of 'productive' infection, but only extremely low numbers of these have 'abundant' expression of unspliced HIV-1 mRNA. These cells do not typically support highly productive HIV-1 infection either in vivo or in vitro. Neurons and glia in vitro support a 'restricted' infection distinguished by often transient production of a low level of virus and viral structural proteins with a relative abundance of viral regulatory mRNAs and proteins (Blumberg et al, 1994). This type of infection may be distinguished, however, from 'latent' infection in which proviral DNA is integrated into the host genome and viral mRNA and structural proteins are not produced.

Both non-transformed and transformed (immortalized) astrocyte and neuronal cell cultures have been reported to support 'restricted' HIV-1 infection (Brack-Werner et al, 1992; Truckenmiller et al, 1993; Ensoli et al, 1994; 1995; Kohleisen et al, 1995; McCarthy et al, 1998a; Nath et al, 1995). These infections are not cytopathic, and they can vary with the strain of HIV-1 (McCarthy et al, 1998a). There is limited production of virus (typically hundreds of pg p24/ml per $10^{6}$ cells), and it is often transient. This contrasts with the infection of human microglia in vitro, where there is sustained production of $\mathrm{ng} / \mathrm{ml}$ quantities of $\mathrm{p} 24$ (Lee et al., 1993; Ionadiss et al, 1995). Restricted infection of neurons or glia in vitro has some capacity for re-activation by inflammatory cytokines (TNF- $\alpha$, IL-1 $\beta$ ) or by co-cultivation with peripheral blood mononuclear cells or T-cells (Tornatore et al, 1991).

The molecular features of restricted HIV-1 infection of glia and neurons in vitro have been studied with cells transfected with infectious proviral DNA, which bypasses restrictions posed by cell binding and entry, and resulted in higher levels of p24 production by these cells (Tornatore et al, 1991; 1994b; McCarthy et al, 1998a,b; Ensoli et al, 1997). The predominant species of mRNA in these cells are 
the multiply-spliced $2 \mathrm{~kb}$ species associated with regulatory gene expression, particularly nef. There is a scarcity of larger molecular weight species associated with structural gene expression. There is a spectrum of post-entry, strain-associated variability in infectivity for neuronal or astrocytic cells (Ensoli et al, 1997; McCarthy et al, 1998a). Recent reports indicate that regulatory elements of the viral LTR are differentially utilized according to cell type, and this could account for differences in the HIV-1 life cycle in neuroepithelial-lineage cells (Ensoli et al, 1997; McCarthy et al, 1998a). Recent reports indicate that regulatory elements of the viral LTR are differentially utilized according to cell type, and this could account for differences in the HIV-1 life cycle in neuroepithelial-lineage cells (Ensoli et al, 1997; McCarthy et al, 1998b). The molecular basis of restricted infection of neurons or glia may lie with the function of rev and/or nef genes. Restricted infection in astrocytes resembles a Rev-deficient phenotype in that there is a lack of efficient synthesis and nuclear export of mRNA species coding for viral structural proteins (Neumann et al, 1995). However, over-expression of nef protein has been reported in astrocytes both in vivo and in vitro (Tornatore et al, 1994a; Saito et al, 1994; Gorry et al, 1998). In vitro, the nef gene sequence and the nef protein may have opposing effects on HIV-1 replication (Gorry et al, 1998). The restricted HIV-1 infection of neurons and glia in vitro may be a relevant though contentious model for HIV-1 infection of neuroepithelial lineage cells in the central nervous system.

\section{References}

Bagasra O, Lavi E, Bobroski L, Khalili K, Pestaner JP, Tawadros R, Pomerantz RJ (1996). Cellular reservoirs of HIV-1 in the central nervous system of infected individuals: identification by the combination of in situ polymerase chain reaction and immunohistochemistry. AIDS 10: 573-585.

Blumberg BM, Gelbard HA, Epstein LG (1994). HIV-1 infection of the developing nervous system: central role of astrocytes in pathogenesis. Virus Res 32: 253-267.

Brack-Werner R, Kleinschmidt A, Ludvigsen A, Mellert W, Neumann M, Herrmann R, Khim MC, Burny A, MullerLantzsch N, Stavrou D (1992). Infection of human brain cells by HIV-1: restricted production in chronically infected human glial cell lines. AIDS 6: $273-285$.

Ensoli F, Ensoli B, Thiele CJ (1994). HIV-1 gene expression and replication in neuronal and glial cell lines with immature phenotype: effects of nerve growth factor. Virology 200: $668-676$.

Ensoli F, Cafaro A, Fiorelli V, Vannelli B, Ensoli B, Thiele CJ (1995). HIV-1 infection of primary human neuroblasts. Virology 210: 221-225.

Gorry P, Purcell D, Howard J, McPhee (1998). Restricted HIV-1 infection of human astrocytes: potential role of nef in the regulation of virus replication. J NeuroVirol 4: 377-386.

Ioannidis JPA, Reichlin S, Skolnik Pur $\alpha$ (1995). Long-term productive human immunodeficiency virus-1 infection in human infant microglia. Am J Pathol 147: 1200-1206.

Kohleisen B, Neumann M, Herrmann R, Brack-Werner R, Krohn KJE, Ovod V, Ranki A, Volker E (1995). Cellular localization of Nef expressed in persistently HIV-1-infected low-producer astrocytes. AIDS, 6: 1427-1436.
Lee SC, Hatch WC, Liu W, Kress Y, Lyman WD, Dickson DW (1993). Productive infection of human fetal microglia by HIV1. Am J Pathol 143: 1032-1039.

McCarthy M, He J, Wood C (1998a). HIV-1 strain-associated variability in infection of primary neuroglia. J NeuroVirol 4: $80-89$.

McCarthy M, Auger D, He J, Wood C (1998b). Cytomegalovirus and human herpesvirus-6 trans-activate the HIV-1 lons terminal repeat via multiple response regions in human fetal astrocytes. I NeuroVirol 4: 495-511.

Nath A, Hartloper V, Furer M, Fowke KR (1995). Infection of human fetal astrocytes with HIV-1: viral tropism and the role of cell to cell contact in viral transmission. J Neuropath Exp Neurol 54: $320-330$

Neumann M, Felber BK, Kleinschmidt A, Froese B, Erfle V, Pavlakis GN, Brack-Werner R (1995). Restriction of human immunodeficiency virus type 1 production in a human astrocytoma cell line is associated with a cellular block in Rev function. J Virol 69: 2159-2167.

Saito Y, Sharer LR, Epstein LG, Michaels J, Mintz M, Louder M, Golding K, Cvetkovich TA, Blumberg BM (1994). Overexpression of nef as a marker for restricted HIV-1 infection of astrocytes in postmortem pediatric central nervous tissues. Neurology 44: $474-480$.

Tornatore C, Chandra R, Berger JR, Major EO (1994a). HIV-1 infection of subcortical astrocytes in the pediatric central nervous system. Neurology 44: 481-487.

Tornatore C, Meyers K, Atwood W, Conant K, Major EO (1994b). Temporal patterns of human immunodeficiency virus type 1 transcripts in human fetal astrocytes. J Virol 68: 93-102.

Tornatore C, Nath A, Amemiya K, Major EO (1991). Persistent human immunodeficiency virus type 1 infection in human fetal glial cells reactivated by T-cell factor(s) or by the cytokines tumor necrosis factor alpha and interleukin-1 beta. J Virol 65: 6094-6100.

Truckenmiller ME, Kulaga H, Coggiano M, Wyatt R, Snyder SH, Sweetman PM (1993). Human cortical neuronal cell line: a model for HIV-1 infection in an immature neuronal system. AIDS Res Hum Retro 9: 445-453.

\section{Molecular dissection of HIV-associated neurodegenerative pathways in transgenic models}

Lennart Mucke

Gladstone Institute of Neurological Disease and

Department of Neurology, University of California, San

Francisco, California 94141-9100, USA

The etiology of HIV-associated dementia appears to be multifactorial involving diverse microbial and host-derived molecules. To characterize the neuropathogenic potential of HIV-1 proteins, HIV-1 receptors and cytokines, we have expressed these molecules in the central nervous system (CNS) of transgenic mice and analyzed the brains of the resulting experimental models at the molecular, histological, and/or functional level. Regulatory sequences of the mouse glial fibrillary acidic protein (GFAP) gene and of the human CD4 (hCD4) gene were used to target the expression of transgenes to astrocytes or microglia, respectively. Glial expression of HIV-1 gp120 or Nef resulted in distinct patterns of neurodegeneration. In the context of immune challenges, astroglial overexpression of transforming growth factor- $\beta 1$ facilitated 
the invasion of the CNS by monocytes, while microglial hCD4 expression mediated a subacute form of neurodegeneration. We are now using tetracycline-regulatable promoters to gain better temporal control over the expression of transgene products and to investigate the reversibility of HIVassociated CNS impairments.

\section{HIV viral proteins are the ammunition that trigger AIDS dementia}

A Nath, M Jones and C Anderson

Departments of Neurology, Microbiology and

Immunology, University of Kentucky, Lexington, Kentucky, USA

The central problem in acquired immunodeficiency syndrome is the profound depletion of CD4-T cells in the blood and neurons in the brain. Even though the virus infects CD4-T cells and rarely infects neurons, cell loss and apoptosis occur predominantly in the uninfected or 'bystander' cells. Several proteins (gp120, gp41, Tat, Nef, Rev and Vpr) encoded by the HIV genome have been shown to cause functional impairment and toxicity to brain cells (Nath and Geiger, 1998) and some of these proteins (gp120 and Tat) also cause lymphocyte toxicity (Oyaizu and Pahwa, 1995). These proteins enter the extracellular compartment either following rupture of HIV infected cells or active release from chronically infected cells. Entry to the brain may also be gained by crossing a disrupted blood brain barrier. Several of these proteins can be detected in the brain, cerebrospinal fluid and serum of HIV-infected patients. The effects of gp120 and Tat on brain function have been studied in some detail. Both proteins cause glial cell activation, production of cytokines, alter glutamate transport, and cause neurotoxicity via excitatory amino acid receptor activation. However, several important differences in subcellular mechanisms triggered by these proteins do occur. Tat and Vpr can cause electrophysiological changes in neurons without causing cell death. These effects are dose responsive and higher dosages can cause irreversible changes leading to neuronal apoptosis. Tat acts directly on the neuronal membrane and action potentials are generated within seconds of exposure, while Vpr causes depolarization only after prolonged exposure of several minutes (Magnuson et al, 1995; Piller et al, 1998). Tat can also cause chemokine (monocyte chemoattractant protein-1) production in glial cells and thus may play an important role in monocyte recruitment to the brain. Tat and gp120 can also induce cytokine production in glial cells (Nath and Geiger, 1998). Tat is even more potent than lipopolysaccharide in inducing tumor necrosis factor- $\alpha$ in monocytes. gp120, gp41 and Tat cause oxidative damage to brain cells and production of nitric oxide (Nath and Geiger, 1998). These proteins cause cell death in only selected populations of neurons. The neurotoxic effects of gp120 and Tat are synergistic. Subtoxic concentrations of Tat and gp120 when incubated together caused neuronal cell death, tentimes lower concentrations were required when compared with responses to the individually applied proteins. An exposure of only a few seconds was sufficient to cause neuronal cell death, but maximal levels of cell death were observed with application lasting $30 \mathrm{~min}$. Thus only a transient exposure to brain cells with a threshold concentration of the viral proteins may be sufficient to cause a 'domino effect' on cerebral function. In conclusion, HIV proteins can account for most of the biochemical, physiological, immunological and pathological abnormalities associated with HIV dementia. Hence viral proteins are likely the 'driving force' that cause HIV dementia. Treatment strategies aimed at controlling the production and action of viral proteins will likely lead to better control of HIV dementia.

\section{References}

Magnuson DS, Knudsen BE, Geiger JD, Brownstone RM, Nath A (1995). Human immunodeficiency virus type 1 tat activates non-N-methyl-D-aspartate excitatory amino acid receptors and causees neurotoxicity. Ann Neurol 37: 373-380.

Nath A, Geiger JD (1998). Neurobiological Aspects of HIV infections: neurotoxic mechanisms. Progress in Neurobio 54: $19-33$.

Oyaizu N, Pahwa S (1995). Role of apoptosis in HIV disease pathogenesis. J Clin Immunol 15: 217-231.

Piller SC, Jans P, Gage PW, Jans DA (1998). Extracellular HIV-1 virus protein $\mathrm{R}$ causes a large inward current and cell death in cultured hippocampal neurons: implications for AIDS pathology. Proc Natl Acad Sci 95: 4595-4600.

\section{Restricted HIV-1 infection in vivo - fact or fiction}

LR Sharer

Department of Pathology, New Jersey Medical School, Newark, New Jersey 07103, USA

We previously reported restricted, non-productive infection of astrocytes in HIV-1 infected children at postmortem, with overexpression of the regulatory gene nef. This was determined in two ways: by immunocytochemistry, using nef-specific antibodies; and by in situ hybridization, using a riboprobe that included mainly the nef portion of HIV-1. By contrast, use of both an antibody to the gag structural protein p24 and a commonly used probe that is complementary to the gag, pol and env regions of HIV-1, but which lacks nef sequences, failed to label astrocytes. In general, results of the studies were complementary, with the nef antibody 
and probe labeling astrocytes but not multinucleated cells and macrophages, while the non-nef antibody and probe gave the reverse pattern. Since the publication of our study, other groups have reported similar findings in the brains of adults with AIDS, indicating restricted, non-productive infection of astrocytes, despite the technical limitations inherent in the use of autopsy tissue. The importance of these studies has been bolstered by in vitro studies of HIV-1 infection of astrocytes, which is usually non-productive, with overexpression of regulatory genes. A key, unresolved question is whether non-productive infection of astrocytes in vivo has clinical relevance. In vitro investigations have suggested that astrocytotropism is common in HIV-1 isolates obtained directly from the central nervous system and that there is impaired function of HIV-1-infected astrocytes, for example with regard to glutamate uptake. It is thus likely that some perturbation of normal astrocyte activity also occurs in vivo, when these cells are directly infected by HIV-1.

\section{References}

Brack-Werner R, Erfle V, Ranki A (1997). Significance of restricted HIV expression for HIV neuropathogenesis: Still an unresolved issue. AIDS 11: 251-252.

Ranki A, Nyberg M, Ovod V, Haltia M, Elovaara I, Raininko R, Haapasalo H, Krohn K (1995). Abundant expression of HIV Nef and Rev proteins in brain astrocytes in vivo is associated with dementia. AIDS 9: 1001-1008.

Saito Y, Sharer LR, Epstein LG, Michaels J, Mintz M, Louder M, Golding K, Cvetkovich TA, Blumberg BM (1994). Overexpression of nef as a marker for restricted HIV-1 infection of astrocytes in postmortem pediatric central nervous tissues. Neurology 44: 474-481.

Sharer LR, Canki M, Bentsman G, Gendelman HE, McComb RD, Volsky DJ (1998). Frequent isolation of astrocytotropic HIV-1 from brain tissue (abstract). J Neuropathol Exp Neurol 57: 480.

\section{Oxidative damage, apoptosis and neurotrophic factors in HIV-encephalitis} Clayton A Wiley, Guoji Wang, Cristian L Achim Department of Pathology, University of Pittsburgh School of Medicine Pittsburgh, Pennsylvania 15213, USA

Neurologic damage is commonly observed in AIDS particularly in the terminal stages of disease. Histopathologic studies have shown neuronal loss, synaptic damage, and white matter abnormalities particularly prominent in HIV encephalitis (Masliah et al, 1992). HIV encephalitis is characterized by abundant infection of CNS macrophages and an even more widespread macrophage activation (Achim et al, 1994; Wiley et al, 1998). While many mechanisms have been proposed, no definitive pathogenesis has been proven for either the gray or white matter damage. The chronic character of the neurologic damage seen in AIDS would be consistent with chronic oxidative stress as seen in other neurodegenerative diseases (Beal, 1996). Reactive oxygen and nitrogen species can mediate neuroglial damage through lipid-peroxidation, DNA strand breakage, enzyme inactivation, and glycooxidation. We examined the extent and distribution of DNA damage associated with HIV-encephalitis. Free $3^{\prime}$ nucleic acids were found in both HIV encephalitis and controls, however, we found no consistent evidence of apoptosis. Since nicked DNA can artifactually develop during tissue processing, we looked for the presence of cellular DNA repair to confirm in vivo significance of DNA damage. Immunocytochemical stains for factors associated with DNA polymerase activity (KU-80) (Love et al, 1998) demonstrated abundant nuclear staining in both control and HIV encephalitis cases. However, KU80 immunocytochemical staining in control tissues was less intense and strictly confined to the nucleus, while KU80 staining in HIV encephalitis was more intense within nuclei and also involved cytoplasm of neuroglial elements. Regions showing nucleic acid damage also exhibited aberrant neutrophic factor expression (Soontornniyomkij et al, 1998). In the basal ganglia of cases with severe HIV encephalitis, BDNF in afferent neocortical projections was decreased while activated macrophages showed increased expression. Chronic oxidative damage to neuroglial cells during HIVencephalitis may be mediated by factors released from activated macrophages or withdrawal of normal neurotrophic support.

\section{References}

Achim CL, Wang R, Miners DK, Wiley CA (1994). Brain viral burden in HIV-infection. J Neuropathol Exp Neuro 53: 284294.

Beal MF (1996). Mitochondria, free radicals, and neurodegeneration. Current Opinion in Neurobio 6: 661-666

Love S, Barber R, Wilcock GK (1998). Apoptosis and expression of DNA repair proteins in ischaemic brain injury in man. Neuroreport 9: 955-959.

Masliah E, Achim CL, Ge N, DeTeresa R, Terry RD, Wiley CA (1992). Spectrum of human immunodeficiency virus-associated neocortical damage. Ann Neurol 32: 321-329.

Soontornniyomkij V, Wang G, Pittman CA, Wiley CA, Achim CL (1998). Expression of brain-derived neurotrophic factor protein in activated microglia of human immunodeficiency virus type 1 encephalitis. Neuropathol App Neurobio 24: $453-460$

Wiley CA, Soontornniyomkij V, Radhakrishnan L, Masliah E, Mellors J, Herman SA, Dailey P, Achim CL (1998). Distribution of brain HIV load in AIDS. Brain Pathol 8: 277-284. 


\section{Co-infections in HIV pathogenesis and neuropathogenesis}

Charles Wood and Micheline McCarthy

University of Nebraska, Department of Biology Science, Lincoln, NE and University of Miami School of Medicine, Miami, Florida, USA

Co-infections of different viruses in the same individuals are frequently observed, especially in AIDS patients. Potential consequences of such coinfection and interaction between co-infecting viruses include the exacerbation of the disease, changing of the infection course, enhancement of viral replication, alteration of target cells, increased virulence and pathogenesis of the infecting viruses. Therefore, an understanding of the mechanism on how viruses interact is important.

Herpesviruses have long been implicated as a cofactor for HIV infection. Most HIV infected individuals are also infected with different herpesviruses. One of them, the human herpesvirus-6 (HHV-6) infects the same host range as HIV, including CD4+ T-cells. It has been demonstrated that co-infection of T-cells by both HIV and HHV-6 leads to increased HIV replication and an enhanced cytopathic effect. This increase is due to the direct transactivation of HIV transcription by HHV-6 gene products in the co-infected cells. In addition, an indirect effect to enhance HIV replication has been observed. We have identified several HHV-6 gene products that can stimulate HIV replication. One of them is a potent inducer of TNF- $\alpha$; stimulation of cytokines secretion upon HHV-6 infection can indirectly activate HIV replication.

Enhancement of viral replication upon co-infection with herpesvirus and HIV can exist bidirectionally. HIV in return can stimulate herpesvirus replication, and could potentially reactivate herpesvirus from latency. One potential mechanism of such activation could be via HIV Tat. Tat can be secreted from infected T-cells, which in turn could activate herpesvirus replication directly or indirectly through cellular factors. We have observed such an effect of Tat in activating HHV-6 replication. In addition, we have observed that another herpesvirus, the Kaposi's sarcoma associated herpesvirus, or HHV-8 can also be stimulated by Tat. Tat was found to stimulate the expression of an early HHV-8 gene, which then stimulated the replication of $\mathrm{HHV}-8$.

Herpesviruses, such as HHV-6, have also been linked to different neurological diseases and may potentially play a role in HIV neuropathogenesis. We have found that HHV-6 can produce a lytic infection of primary astrocytes in culture, and can potentiate the replication of HIV in astrocytes. It is likely that HHV-6 infection will alter cellular factors and cytokine gene expression, as well as astrocyte function. It may also indirectly affect HIV infection of other neural cells, including the microglia cells.

\section{Acknowledgements}

This symposium was sponsored by the National Institute for Neurological Disorders and Stroke and by the Department of Pathology, University of Miami School of Medicine and organized by Dr AP Kerza-Kwiatecki (NINDS) and Dr Carol K Petito (Miami). Ms Linda Hung provided expert administrative assistance and support.

\section{NINDS officials}

Dr FJ Brinley, Jr, Director, Division of Convulsive Infectious and Immune Disorders. National Institute of Neurological Disorders and Stroke, National Institutes of Health, Bethesda, Maryland, USA.

Dr AP Kerza-Kwiatecki, Program Director, National Institute of Neurological Disorders and Stroke, National Institutes of Health, Bethesda, Maryland, USA.

Dr AS Penn, Deputy Director, National Institute of Neurological Disorders and Stroke, National Institute of Health, Bethesda, Maryland, USA.

\section{Invited participants}

Dr E Benveniste, Professor of Cell Biology, Department of Cell Biology, University of Alabama at Birmingham, Birmingham, Alabama, USA.

Dr LH Boise, Assistant Professor, Department of Microbiology and Immunology, University of Miami School of Medicine, Miami, Florida, USA.

Dr VL Dawson, Associate Professor of Neurology, Neuroscience \& Physiology, Department of Neurology, Johns Hopkins University School of Medicine, Baltimore, Maryland, USA.

Dr HE Gendelman, David T Purtillo Distinguished Professor of Pathology and Microbiology, University of Nebraska Medical Center, Omaha, Nebraska, USA.

Dr MA Goldsmith, Assistant Investigator, Gladstone Institute of Virology and Immunology, Assistant Professor of Medicine, UCSF, San Francisco, California, USA.

Dr K Khalili, Director and Professor, Center for Neurovirology and NeuroOncology, MCP Hahnemann University, Philadelphia, Pennsylvania, USA. 
Dr AA Lackner, Associate Professor, Harvard Medical School of Medicine, Chairman, Division of Comparative Pathology, New England Regional Primate Research Center, Southborough, Massachusetts, USA.

Dr SA Lipton, Chief, CNS Research Institute, Harvard Medical School, Brigham and Women's Hospital, Boston, Massachusetts, USA.

Dr E Masliah, Professor of Neurosciences and Pathology, University of California School of Medicine, La Jolla, California, USA.

Dr MP Mattson, Professor of Anatomy and Neurobiology, Associate of the Sanders-Brown Research Center on Aging, University of Kentucky, Lexington, Kentucky, USA.

Dr JC McArthur, Professor of Neurology and Epidemiology, Department of Neurology, Johns Hopkins University School of Medicine, Baltimore, Maryland, USA.

Dr M McCarthy, Associate Professor of Neurology, Department of Neurology, VA Medical Center, University of Miami School of Medicine, Miami, Florida, USA.

Dr L Mucke, Director, Gladstone Institute of Neurological Disease, Professor of Neurology, UCSF, San Francisco, California, USA.
Dr O Narayan, Professor and Chairman, Department of Microbiology, Molecular Genetics and Immunology, Marion Merrell Dow Foundation Distinguished Professor, University of Kansas, Kansas City, Kansas, USA.

Dr A Nath, Associate Professor, Department of Neurology, University of Kentucky, Lexington, Kentucky, USA.

Dr CK Petito, Professor of Pathology, University of Miami School of Medicine, Miami, Florida, USA.

Dr ER Podack, Professor and Chairman, Department of Microbiology and Immunology, University of Miami School of Medicine, Miami, Florida, USA.

Dr P Shapshak, Research Professor, Department of Psychiatry and Behavioral Sciences, University of Miami School of Medicine, Miami, Florida, USA.

Dr LR Sharer, Professor of Pathology, Department of Pathology, New Jersey Medical School New Jersey, Newark, New Jersey, USA.

Dr CA Wiley, Professor of Pathology, Department of Pathology, Director Division of Neuropathology, University of Pittsburgh, Pittsburgh, Pennsylvania, USA.

Dr C Wood, Lehr/3M Professor of Biological Sciences, University of Nebraska School of Biological Sciences, Lincoln, Nebraska, USA. 